\title{
Torsion of cryptorchid testis after primary orchiopexy failure: An overlooked cause of abdominal pain in children
}

\author{
Aniruddha Ghosh ${ }^{1}$, *Soumya Roy $^{2}$, Arunaloke Bhattacharya ${ }^{3}$, Maya Mukhopadhyay ${ }^{4}$, Jaydeep Choudhury ${ }^{3}$ \\ Sri Lanka Journal of Child Health, 2019; 48(1): 82-83 \\ DOI: http://dx.doi.org/10.4038/sljch.v48i1.8660 \\ (Key words: Orchiopexy, children, abdominal pain, torsion of testis, testicular gangrene, cryptorchid testis)
}

\begin{abstract}
Introduction
Abdominal pain for more than two weeks is complained of by more than one third of all children ${ }^{1}$. However, acute abdominal pain requiring surgical intervention is found in about $1 \%$ of all children presenting to the emergency department. It is a diagnostic challenge to timely recognise them, because of the subtle clinical signs. Hence many children suffer the consequences of late diagnosis ${ }^{2}$. Testicular torsion as a cause of acute abdominal pain is well known but torsion of the cryptorchid testes continues to elude the clinicians from making a timely diagnosis $^{3}$. It may be noted that failure of orchiopexy and subsequent return of the testis from the scrotum to the intra-abdominal position is well documented in the literature 4 . We report the case of young boy with a history of orchiopexy one year back, who presented with intense abdominal pain. It was finally diagnosed that the primary orchiopexy had failed and the testis had reverted to the intra-abdominal position, where it had undergone torsion and eventual gangrene. To the best of our knowledge, this is the first case reporting such a cause of abdominal pain in children.
\end{abstract}

\section{Case report}

A 5 year and 9 month old male child was admitted with a complaint of abdominal pain for the last five days. Pain had increased over the last two days but was not associated with any vomiting, constipation, diarrhoea, dysuria or fever. He had a history of left sided orchiopexy one year back due to testicular torsion.

\footnotetext{
${ }^{1}$ Resident of Paediatrics, ${ }^{2}$ Postgraduate Trainee in Paediatrics, ${ }^{3}$ Associate Professor of Paediatric Medicine, ${ }^{4}$ Professor of Paediatrics, Institute Of Child Health, Kolkata, India

*Correspondence: dr.roy85@gmail.com (Received on 23 April 2017: Accepted after revision on 16 June 2017)

The authors declare that there are no conflicts of interest

Personal funding was used for the project.

Open Access Article published under the Creative
}

Commons Attribution CC-BY

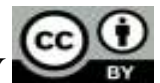

General examination and vital parameters were within normal limits. There was diffuse tenderness in the left iliac fossa (LIF). However, no mass was palpable in the LIF. The abdomen was soft, no organomegaly was palpable and intestinal peristaltic sounds were well auscultated.

Initial tests showed normal blood counts, C-reactive protein, electrolytes, hepatic and renal parameters. Serum amylase and lipase, urine routine examination as well as culture and stool examination were noncontributory. Treatment was presumptively started with pantoprazole and drotaverine but pain showed no improvement. An ultrasonogram of the abdomen showed an ill-defined hypoechoic focus $(1.4 \mathrm{~cm} \times 2.2$ $\mathrm{cm}$ ) in the LIF. Magnetic resonance imaging (MRI) of the abdomen revealed a round hyperintense lesion (1.3 $\mathrm{cm} \times 1.3 \mathrm{~cm}$ ) lateral to the sigmoid colon on fat suppressed images. Ill-defined hyperintensities were noted around it extending into the left inguinal canal. The rounded structure on MRI appeared to be an intraabdominal left testis with oedema around; furthermore, the left scrotal sac showed no testis like structure on MRI (Figure 1).

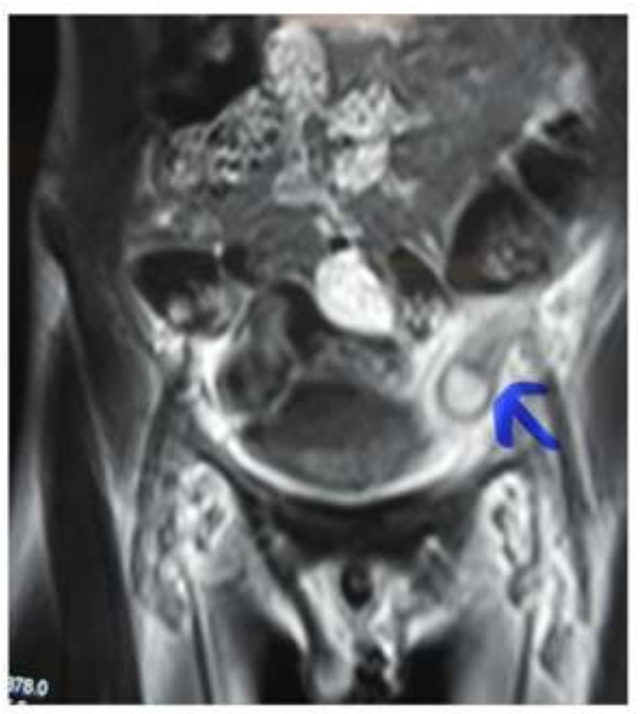

Figure 1: MRI abdomen showing the left testis in intra-abdominal position 
An orchiopexy failure was contemplated and immediate laparotomy was arranged. During surgery, the left testis was found to be in the abdomen lateral to sigmoid colon with torsion. It was in a gangrenous state, which prompted the surgeon to do a left-sided orchiectomy. Postoperative biopsy confirmed the mass to be a necrotic testis with features of ischaemia and no malignant changes. Pain resolved immediately after surgery and child was discharged after five days.

\section{Discussion}

The failure rate of primary inguinal orchiopexy has been reported to be from 7.5 to $13 \%{ }^{4}$. McIntosh et al. reported that the failure rate of primary orchiopexy over an eighteen year-long study was only $1.6 \%$ for unilateral orchiopexy and $1.9 \%$ for bilateral orchiopexy (two testes operated in two different settings). However, when bilateral orchiopexy was done in same setting, the failure rate increased slightly to $1.9 \%$ per testicle ${ }^{5}$. Ziylan et al. found that reoperation (secondary orchiopexy) had to be done after a mean duration of 3.2 years after the initial orchiopexy and that the mean age of presentation for secondary operation was 6.8 years. They also reported that the testis (after primary orchiopexy) was displaced to high scrotal position in $46.9 \%$, at the external ring in $25 \%$ and within the inguinal canal in $28.1 \%$ patients ${ }^{4}$. Our patient was slightly younger (5 years and 9 months of age) with a history of unilateral orchiopexy one year before. His testis was found to be located in the abdomen. Inadequate mobilization of the testis, failure to perform true high ligation of the hernia and unsuccessful fixation of testis into scrotum are some of the important causes ${ }^{4}$. McIntosh et al. found that bilateral orchiopexy and older age during surgery were two risk factors for failure of orchiopexy. When the primary operation was done at $<2$ years of age, failure was $1.97 \%$ whereas at 2 to 6 years of age, it increased to $2.67 \%$. Above 6 years, it was $0-0.8 \%{ }^{5}$.

Pogorelic Z et al. evaluated eight patients with torsion of testis in the inguinal canal (All were naive cases, unlike our case who had a failed primary orchiopexy). Four children, in whom testis preserving orchiopexy could be performed, had presented to the surgeon with a mean duration of symptoms of 6 hours. The remaining four children, in whom orchiectomy had to be done for gangrene of testis, had a mean duration of presentation of 50 hours $^{6}$. This highlights the paramount importance of early clinical suspicion, because re-orchiopexy (secondary orchiopexy), though a difficult surgical procedure, nevertheless has the success rate of as high as 81 to $100 \%{ }^{1}$. Our case had symptoms for 5 days before presentation and quite expectedly, had irreversible gangrene of the testis, needing orchiectomy.

This case report highlights the importance of examining the genitalia during an abdominal examination in male children especially if the child has abdominal pain. The past history of orchiopexy should have made this imperative. If this was done during the initial abdominal examination, the absence of the testis in the scrotum would have been detected and the true diagnosis suspected much earlier.

\section{References}

1. Lake AM. Chronic abdominal pain in childhood: diagnosis and management. American Family Physician 1999; 59(7):1823-30.

2. Kim JS. Acute abdominal pain in children. Pediatric Gastroenterology, Hepatology \& Nutrition 2013; 16(4): 219-24.

3. Moore CP, Marr JK, Huang CJ. Cryptorchid testicular torsion. Pediatric Emergency Care 2011; 27(2):121-3.

4. Ziylan O, Oktar T, Korgali E, Nane I, Ander H. Failed orchiopexy. Urologia Internationalis 2004; 73(4):313 -5.

5. McIntosh LA, Scrimgeour D, Youngson CG, Driver CP. The risk of failure after primary orchidopexy: An 18 year review. Journal of Pediatric Urology 2013; 9(6 Pt A):759-62.

6. Pogorelić Z, Mrklić I, Jurić I, Biočić M, Furlan D. Testicular torsion in the inguinal canal in children. Journal of Pediatric Urology 2013; 9(6 Pt A):793-7. 